\title{
Multi-state analysis of hypertension and mortality: application of semi-Markov model in a longitudinal cohort study
}

\author{
Azra Ramezankhani ${ }^{1}$, Michael J. Blaha ${ }^{2}$, Mohammad hassan Mirbolouk ${ }^{2}$, Fereidoun Azizi ${ }^{3}$ and Farzad Hadaegh ${ }^{1 *}$ (D)
}

\begin{abstract}
Background: Most previous research has studied the association of hypertension with cardiovascular disease (CVD) and all-cause mortality by focusing on the transition from the initial state to a single outcome. We investigated the impact of hypertension, defined according to the 2017 American College of Cardiology/American Heart Association (ACC/AHA) (new) and the Seventh Report of the Joint National Committee (JNC7) (old), on CVD death and all-cause mortality considering non-fatal CVD as an intermediate event between two CVD-free and mortality states.

Methods: A total of 3002 Iranian population (47.4\% men), aged $\geq 50$ years were followed from 1999 to 2014. Two multi-state semi-Markov models with three transitions were defined for CVD death and all-cause mortality as two outcomes. The multivariable Cox model was used to estimate the effect of hypertension on transition hazards. The mean of 15-year life expectancy of participants in each transition was estimated using the restricted mean survival time.

Results: The ACC/AHA guideline increased the prevalence of hypertension from 43.3 to 68.6\%. Among CVD-free individuals, hypertension was significantly associated with increased risk of non-fatal CVD [Hazard Ratio, 1.52 (1.28-1.81) and 1.48 (1.21-1.80)], CVD death [2.96 (2.06-4.25) and 1.98 (1.30-3.04)] and all-cause mortality [1.64 (1.32-2.05) and 1.31 (1.01-1.69)] according the old and new guidelines, respectively. However, after incident non-fatal CVD, the association between hypertension and mortality events was not significant according to both definitions. Hypertensive participants experienced a first non-fatal CVD about 0.9 and 0.6 years earlier than normotensive population according to JNC7 and the 2017 ACC/AHA guidelines, respectively.

Conclusion: Hypertension, according to JNC7 and the ACC/AHA guidelines, significantly increased the risk of mortality events among CVD-free population although the risk was attenuated using ACC/AHA guideline. Hypertension also decreased the number of years lived without CVD and early onset of CVD, and consequently, an increase in the time spent with these diseases. After non-fatal CVD, hypertension had no significant impact on mortality risk according to both guidelines.
\end{abstract}

Keywords: Hypertension, Guideline, Multi-state, Mortality, Cardiovascular

\footnotetext{
* Correspondence: fzhadaegh@endocrine.ac.ir

${ }^{1}$ Prevention of Metabolic Disorders Research Center, Research Institute for Endocrine Sciences, Shahid Beheshti University of Medical Sciences, Tehran, Iran

Full list of author information is available at the end of the article
} 


\section{Background}

By 2030, almost 23.6 million people will die of cardiovascular disease (CVD), mainly from coronary heart disease (CHD) and stroke [1]. There has been a wellestablished relation between hypertension and CVD as well as premature death [2, 3]. Considerable evidence exists about the impact of hypertension on the risk of CVD and mortality among CVD-free individuals [2, 4]. Moreover, a great number of studies has shown the effect of hypertension on recurrent CVD and all-cause mortality among individuals with history of CVD [5, 6]. The majority of these studies have focused on the transition from the initial state to a single outcome; however, patients may experience several events in the path between two initial and end points. For example, an increased risk of death among hypertensive patients after incident CVD has been evaluated without considering CVD as an intermediate event that take place "between initial condition and the endpoint". Analysis in such studies is often performed using multi-state models (MSM) [7, 8]. In a MSM, a number of states are defined and the focus is on the process of going from one state to another [7, 9]. A widely used MSM, known as the illness-death model, can be used to evaluate whether previously diseased individuals have the same rate of death as those who have been healthy all their lives $[8,10,11]$. These models can provide a detailed insight into the effects of exposures on each state.

Recently, the American College of Cardiology/American Heart Association (ACC/AHA) [12] have proposed lower thresholds $(>130 / 80 \mathrm{mmHg}$ ) for blood pressure (BP) to define hypertension relative to prior guidelines $(>140 / 90$ $\mathrm{mmHg}$ ) [13]. Therefore, using the $2017 \mathrm{ACC} / \mathrm{AHA}$ and the Seventh Report of Joint National Committee (JNC7) criteria to define prevalent cases of hypertension, we undertook this study to investigate how MSM can be applied to study the effect of different definition for hypertension on the risk of CVD death and all-cause mortality with and without non-fatal CVD. We developed two MSMs each with three states: CVD-free (state 1), non-fatal CVD (state 2) and mortality events (state 3). In analysis of multi-state data, we focused on four topics: 1) the impact of hypertension on the probabilities of non-fatal CVD,CVD death and allcause mortality among individuals free of CVD at baseline, 2) the probability of all-cause mortality and CVD death after non-fatal CVD occurrence, 3) life expectancy (LE) of participants in different states and comparing it among the participants with and without hypertension, and 4) the number of years of life lost due to hypertension with and without non-fatal CVD. To accomplish these goals, we analysed data from Tehran Lipid and Glucose Study (TLGS).

\section{Methods}

\section{Participants}

TLGS is an ongoing follow-up study of Iranian population with the goal of determining the risk factors and outcomes for non-communicable diseases [14]. Briefly, in phase 1 (1999-2001), about 15,000 individuals aged $\geq 3$ years participated, and a total of 3550 new subjects were included in phase 2 (2002-2005). Upon entering the study, more examinations were conducted every 3 years. Besides the triennial reexaminations, all participants were followed up annually by telephone call about any medical event leading to hospitalization during the past year.

In this study, all subjects aged $\geq 50$ years $(n=3890)$ from the first and second phases were included. Participants with prevalent CVD at baseline $(n=502)$, with no information on hypertension status $(n=70)$ and without any follow-up data $(n=316)$ were excluded. The remaining 3002 (1422 men) participants were followed for different outcomes including non-fatal CVD and death from any cause (CVD and non-CVD death) until the end of the study (20 March 2014) (Fig. 1). The ethics committee of the Research Institute for Endocrine Sciences of Shahid Beheshti University of Medical Sciences approved the study and informed written consent was obtained from all participants.

\section{Data collection}

In all examinations, participants completed a questionnaire on age, sex, history of CVD, medication use and smoking habits. Anthropometric measurements were also made, including height, and weight. After a 15-min rest in the sitting position, two measurements of $\mathrm{BP}$ were taken at the right brachial artery; the mean of two measurements was used to define systolic blood pressure (SBP) and diastolic blood pressure (DBP). Blood samples were obtained after a 12-h overnight fast to assess the fasting plasma glucose (FPG) and total cholesterol (TC) using standard laboratory techniques [14].

\section{Exposure}

In all examinations, hypertension was defined as a $\mathrm{SBP} \geq$ $140 \mathrm{mmHg}$ or a DBP $\geq 90 \mathrm{mmHg}$ or taking antihypertensive medications according to the JNC7 guideline [13], and SBP $\geq 130 \mathrm{mmHg}$ or a $\mathrm{DBP} \geq 80 \mathrm{mmHg}$ or taking antihypertensive medications in accordance with 2017 ACC/AHA guideline [12].

\section{Confounding variables}

We considered multiple potential confounders based on our previous study [15]. They included age, sex, TC, body mass index (BMI) calculated as weight $(\mathrm{kg}) / \mathrm{height}$ $\left(\mathrm{m}^{2}\right)$, smoking status (current, former/never), and diabetes mellitus (defined as $\mathrm{FPG} \geq 7 \mathrm{mmol} / \mathrm{L}$ or 2 -h post- 


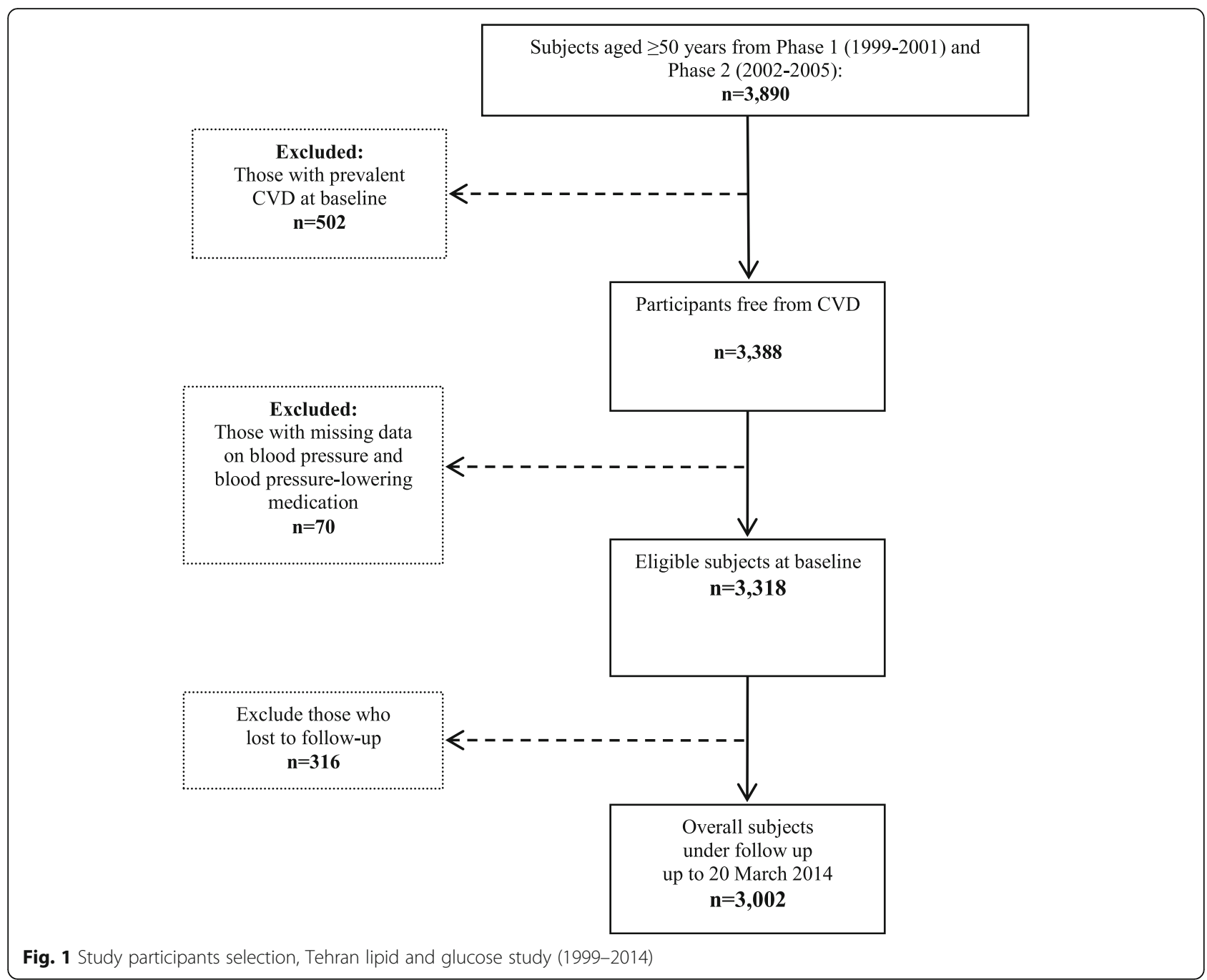

challenge plasma glucose $\geq 11.1 \mathrm{mmol} / \mathrm{L}$ [16] or taking anti-diabetic medications).

\section{Outcome}

Details on the collection of outcomes in TLGS have been presented elsewhere [14, 17]. To summarize, in this study, all participants were followed annually for any medical conditions from entry into the study until the end of the study. The three outcomes for our analyses were incident non-fatal CVD, CVD mortality and all-cause mortality. Non-fatal CVD was defined as definite myocardial infarction (MI), probable MI, unstable angina pectoris, angiographic proven CHD, heart failure and stroke. CVD death was defined as fatal coronary artery diseases, MI, or stroke as either the primary or a contributing cause of death. Allcause mortality was defined as death from all causes including CVD and non-CVD death.

\section{Statistical analysis}

Continuous and categorical variables were compared using the independent sample t-tests and $\chi^{2}$ test, respectively. Incidence density rate of events and respective $95 \%$ confidence interval (CI) were calculated by dividing the number of events by the person-years at risk. Missing data (after the exclusion criteria was applied) ranged from $0 \%$ to $\sim 3 \%$ across the confounders; therefore, the multivariate imputation by chained equations (mice package in $\mathrm{R}$ software) [18] was implemented for handling missing data.

\section{Multi-state model}

We defined two MSMs for our data analysis (Fig. 2). In both MSMs, the state CVD-free was defined as state 1 and non-fatal CVD was defined as state 2. CVD death and all-cause death were defined as states 3 in MSM1 and MSM2, respectively. A change of state is called a 


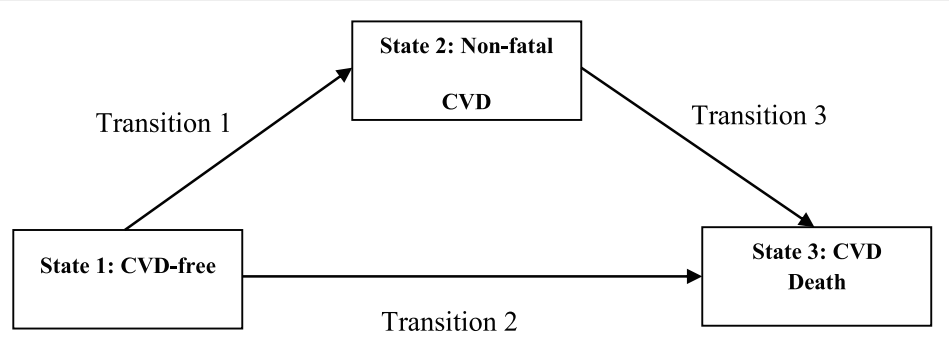

$\mathbf{A}$

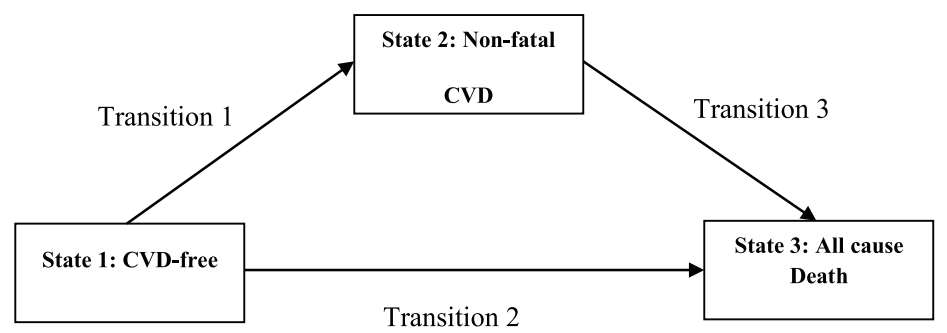

B

Fig. 2 Graphical representation of the two multi-state models, a multi-state model 1 in which state 3 is CVD death, $\mathbf{b}$ multi-state model 2 in which state 3 is all cause deathThe association between hypertension, defined according to the 2017 ACC/AHA and JNC7, on CVD death and allcause death considering non-fatal CVD as an intermediate event between two CVD-free and mortality states was estimated. ACC/AHA: The American College of Cardiology/American Heart Association. CVD: cardiovascular diseases. JNC7: The Seventh Report of the Joint National Committee.

transition. In Fig. 2, boxes represent the states and arrows show the possible transitions. All patients start in state 1 , some of them move to state 2 (transition 1 ) and some patients transit directly to state 3 (transition 2 ). It is also possible to move from state 2 to state 3 (transition 3). State 3 (CVD death/all-cause mortality) is an absorbing state, because no transition can emerge from it [7]. This model is known as illness-death model [19]. The basic quantities of interest in a MSM are the transition intensities or hazard rates. The hazard rate is defined as instantaneous risk of moving from state $r$ to state $\boldsymbol{s}$ at time $\boldsymbol{t}$. Two approaches often used in a MSM for definition of time $\boldsymbol{t}$ in the hazard function. The first approach is "clock forward" in which time $\boldsymbol{t}$ refers to the time since the individuals entered the initial state (start of study). In the second approach, defined as "clock reset", time $\boldsymbol{t}$ refers to the time since entry of the present state. Thus, the clock is reset to 0 every time the patient enters a new state [9]. There are also different probability models to describe the way that an individual moves through a series of states in a multi-state process. A model that is often assumed in practice is the Markov model, which implies that the probability of going to a future state depends only on the present state and not on the history. The Markov property cannot hold when "clock reset" is considered as time scale; because, in this approach future state not only depends on the current state, but also on the entry time into current state. In case of "clock reset", the resulting multi-state model is called a Markov renewal or semi-Markov model, which forms a sequence of embedded Markov models.

Finally, to estimate the hazard rates in a MSM, different functional forms such as parametric, semiparametric or non-parametric approaches can be applied [9]. In our study, we used Cox's proportional hazards model which also allows assessing the effects of multiple covariates on hazard rates. A preliminary analysis in both MSMs indicated independency of transition hazards going out from state 2 on the time at which that state was reached. Hence, a time homogenous semiMarkov model in the framework of Cox model was considered for data analysis.

To accommodate different baseline hazards for each transition, the data were stratified by transitions. If the endpoint of interest (states 2 and 3) had not yet occurred at the end of the observation period (20 March 2014), the event time was defined as right censored. The event time was calculated as the time between baseline examination and the event date (for event cases) or the last follow-up (for censored cases). Participants were 
censored due to death from a cause other than event of interest, loss to follow-up, or the end of the study without the event occurring.

All analysis was performed on a pooled sample wherein gender was included as a covariate in all models. The models were adjusted for age and sex, and were further adjusted for smoking status, TC, diabetes status and BMI. As we used "clock reset" to define the time scale in both MSMs, the time returns to zero at every transition, and the observations start at time zero after each transition. For illness-death models, this issue concerns only the transition from non-fatal CVD to mortality events. Therefore, for transition 1 and 2, the baseline measurements of exposure and covariates were used in MSMs. But, for the transition 3 (non-fatal CVD to mortality events), the most recent available values before incident of non-fatal CVD for each measurement was used. We also compared the magnitude of hazard ratios (HRs) of hypertension for two definitions of hypertension according to JNC7 vs. the 2017 ACC/AHA criteria in each MSM model.

\section{Mean of survival time}

We estimated the mean of 15-year LE of participants in each transition using the restricted mean survival time (RMST), defined as the area under the curve of the survival function up to a truncation time point $\tau(<\infty)$ :

$$
\mu_{t}=\int_{0}^{\tau} S(t) d t
$$

where $S(t)$ is the survival function for the time $T$. The interpretation of $\mu$ in our study with a duration of 15 years is LE in each transition for the next 15 years [20]. The difference between hypertensive patients' RMST and the RMST of the normotensive population was interpreted as the number of years of life lost due to hypertension [21]. We also estimated restricted mean time lost (RMTL) and the ratio of RMTLs for hypertensive and normotensive individuals in each transition. RMTL is defined as the average years of life lost during the follow-up time and is the area above the curve of the survival function up to a time $\mathrm{t}\left(\tau-\mu_{\mathrm{t}}\right)$. All analysis was done in $\mathrm{R}$ (https://CRAN.R-project.org). Unadjusted RMST and RMTL were estimated using survRM2 package and adjusted estimation for RMST were obtained by applying pseudo-value technique proposed by Kleinet al [22] using the pseudo package in R. The multi-state Markov model and the Cox PH model were fitted in $\mathrm{R}$ using the mstate [23] and survival packages, respectively. We used a $P$-value of $<0.05$ (two tailed) to determine statistical significance.

\section{Results}

\section{Descriptive}

The cohort included 3002 participants between the ages of 50 and 88 years. The mean (SD) age of participants was 60.0 (7.5) years where 52\% were women (Table 1). The prevalence of hypertension was 43.3 and $68.6 \%$ according to JNC7 and the 2017 ACC/AHA guidelines, respectively (Table 1). The reverse Kaplan-Meier estimate [24] of the median follow-up was 13.95 (interquartile range 10.29-14.50) years in MSM 1, and 14.09 (12.1114.54) years in MSM 2 . The numbers of transitions, both in terms of frequencies and percentages, are shown in Table 2. In both MSMs, 3002 individuals were at risk of transition from state 1 to states 2 and 3 . Of these, 601(341 men) experienced a non-fatal CVD (transition 1) (Table 2). In MSM1, out of 601 individuals who experienced first non-fatal CVD, 71(51 men) died of CVD (transition 3) and 530 remained alive with non-fatal CVD until the end of the study. Among 3002 individuals free of CVD at baseline, 156 (96 men) died of CVD (transition 2). In MSM 2, from 601 individuals who experienced first non-fatal CVD, 126 (86 men) died of any cause (transition 3) and 475 remained alive with nonfatal CVD until the end of the study. Also, from 3002 individuals at the beginning of the study, 367 (210 men) died of any cause (transition 2) (Table 2).

The estimated cumulative hazards for the 3 transitions in two MSMs have been shown in Fig. 3. In both MSMs, the cumulative hazard is higher in transition 3 for normotensive participants.

\section{Results of MSM1}

The results from MSM 1 are presented in Table 3. In confounders adjusted models, hypertensive individuals had 52 and $48 \%$ increased risk for non-fatal CVD according to JNC7 and the 2017 ACC/AHA guidelines, respectively, compared with their normotensive counterparts. The difference between the confounders adjusted HRs of hypertension according to JNC7 vs. the 2017 ACC/AHA guideline was not significant $(p=0.360)$.

Among 3002 participants free of CVD at baseline, hypertensive participants had 2.96 and 1.98 fold increased risk for CVD death according to JNC7 and the 2017ACC/ AHA guidelines, respectively. Significant differences were found between the confounders adjusted HRs of hypertension according to JNC7 vs. the 2017 ACC/AHA guideline $(p=0.015)$. However, the risk of CVD death after the nonfatal CVD occurrence was not significantly associated with hypertension according to both JNC7 and the 2017 ACC/ AHA guidelines (Table 3).

Table 4 shows the estimations of fifteen-year RMST and RMTL. Hypertensive participants were found to have 0.5 and 0.3 years shorter LE without CVD compared to normotensives according to JNC7 and the 2017 
Table 1 Characteristics of study cohort $(n=3002)$ at baseline according to JNC7 and the 2017 ACC/AHA guidelines for definition of hypertension, Tehran Lipid and Glucose Study (1999-2014)

\begin{tabular}{|c|c|c|c|c|c|c|}
\hline \multicolumn{3}{|c|}{ According to JNC7 } & \multicolumn{3}{|c|}{ According to the 2017 ACC/AHA } & \multirow{2}{*}{$\begin{array}{l}\text { Total } \\
\text { population } \\
\mathbf{N}=3002\end{array}$} \\
\hline $\begin{array}{l}\text { Hypertensive } \\
n=1301\end{array}$ & $\begin{array}{l}\text { Normotensive } \\
n=1701\end{array}$ & P-value & $\begin{array}{l}\text { Hypertensive } \\
n=2060\end{array}$ & $\begin{array}{l}\text { Normotensive } \\
n=942\end{array}$ & $\overline{P \text {-value }}$ & \\
\hline
\end{tabular}

Sex

\begin{tabular}{|c|c|c|c|c|c|c|c|}
\hline Male & $531(40.8)$ & 891 (52.4) & $<0.001$ & $897(43.5)$ & $525(55.7)$ & $<0.001$ & $1422(47.4)$ \\
\hline Female & $770(59.2)$ & $810(47.6)$ & & $1163(56.5)$ & $417(44.3)$ & & $1580(52.6)$ \\
\hline Age (year) & $61.4(7.6)$ & $58.8(7.2)$ & $<0.001$ & $60.5(7.5)$ & $58.9(7.4)$ & $<0.001$ & $60.0(7.5)$ \\
\hline $\mathrm{TC}(\mathrm{mmol} / \mathrm{L})$ & 6.07 (1.28) & $5.77(1.18)$ & $<0.001$ & $6.00(1.25)$ & $5.68(1.17)$ & $<0.001$ & $5.90(1.23)$ \\
\hline SBP $(\mathrm{mmHg})$ & $149.9(19.7)$ & 119.1(11.4) & $<0.001$ & $141.3(20.0)$ & $113.04(9.5)$ & $<0.001$ & $132.4(21.8)$ \\
\hline $\mathrm{DBP}(\mathrm{mmHg})$ & $88.8(11.5)$ & $75.2(7.9)$ & $<0.001$ & $86.01(10.40)$ & $70.46(6.17)$ & $<0.001$ & $81.1(11.7)$ \\
\hline \multirow[t]{2}{*}{ BMI $\left(\mathrm{kg} / \mathrm{m}^{2}\right)$} & $28.8(4.5)$ & $26.9(4.2)$ & $<0.001$ & $28.4(4.4)$ & $26.2(4.2)$ & $<0.001$ & $27.7(4.5)$ \\
\hline & \multicolumn{2}{|c|}{$N(\%)$} & \multicolumn{4}{|c|}{$N(\%)$} & $N(\%)$ \\
\hline \multicolumn{8}{|c|}{ Smoking status } \\
\hline Current & 103(7.9) & 293(17.2) & $<0.001$ & $187(9.1)$ & $209(22.3)$ & $<0.001$ & $396(13.1)$ \\
\hline Never & 1033(79.4) & $1211(71.1)$ & & $1620(78.8)$ & $624(66.5)$ & & $2244(74.7)$ \\
\hline Past & $162(12.4)$ & 192(11.2) & & $248(12.1)$ & $106(11.3)$ & & $354(11.7)$ \\
\hline \multicolumn{8}{|l|}{ Diabetes } \\
\hline Yes & $386(29.6)$ & 294(17.2) & $<0.001$ & $539(27.0)$ & $141(15.5)$ & $<0.001$ & $680(22.6)$ \\
\hline No & $872(67.0)$ & 1353(79.5) & & $1456(73.0)$ & $769(84.5)$ & & $2225(74.1)$ \\
\hline \multicolumn{8}{|c|}{ Antihypertensive medication use } \\
\hline yes & $498(38.3)$ & 0 & & $498(24.2)$ & 0 & & 498(16.6) \\
\hline
\end{tabular}

JNC7, The Seventh Report of Joint National Committee

ACC/AHA, The 2017 American College of Cardiology/American Heart Association

$T C$ total cholesterol, SBP systolic blood pressure, DBP diastolic blood pressure, $B M I$ body mass index

ACC/AHA guidelines, respectively. They also experienced a first non-fatal CVD about 0.9 and 0.6 years earlier than normotensive population according to JNC7 and the 2017 ACC/AHA guidelines. However, after experiencing a first non-fatal CVD, no significant difference in the LE of two hypertensive and normotensive groups was observed according to both JNC7 and the 2017 ACC/AHA guidelines. As shown in Table 4, the average years of life lost (RMTL) was 2.6 and 2.0 times higher in hypertensive vs. normotensive participants according to JNC7 and the 2017 ACC/AHA guidelines, respectively. Table 5 shows the results of confounders adjusted RMST. Hypertensive individuals had the shorter RMST, in transitions 1 and 2, according to both JNC7 and the 2017 ACC/ AHA guidelines.

Table 2 Numbers and percentages of population in the multi-state models; according to JNC7 and the 2017 ACC/AHA guidelines, Tehran Lipid and Glucose Study (1999-2014)

\begin{tabular}{|c|c|c|c|c|c|}
\hline & \multirow[b]{2}{*}{ Origin states } & \multirow[t]{2}{*}{ Total entering } & \multicolumn{3}{|c|}{ Destination states } \\
\hline & & & 1: CVD-free & 2: Non-fatal CVD & 3: CVD death \\
\hline \multirow[t]{3}{*}{ Multi-state model 1} & 1: CVD-free & 3002 & 2245 (74.8\%) & $601(20 \%)$ & $156(5.2 \%)$ \\
\hline & 2: Non-fatal CVD & 601 & - & $530(88.1 \%)$ & 71 (11.9\%) \\
\hline & 3: CVD death & 227 & - & - & $227(100 \%)$ \\
\hline Multi-state & & & 1: CVD-free & 2: Non-fatal CVD & 3: All-cause death \\
\hline \multirow{3}{*}{ model 2} & 1: CVD-free & 3002 & $2034(67.7 \%)$ & $601(20.1 \%)$ & $367(12.2 \%)$ \\
\hline & 2: Non-fatal CVD & 601 & - & $475(79.0 \%)$ & $126(21.0 \%)$ \\
\hline & 3: All-cause death & 493 & - & - & 493 (100\%) \\
\hline
\end{tabular}

In multi-state model 1, the state 3 is CVD death. Numbers and percentages of population in each transition are similar according to both JNC7 and the 2017 ACC/AHA guidelines

In multi-state model 2, the state 3 is all cause-death. Numbers and percentages of population in each transition are similar according to both JNC7 and the 2017 ACC/AHA guidelines 

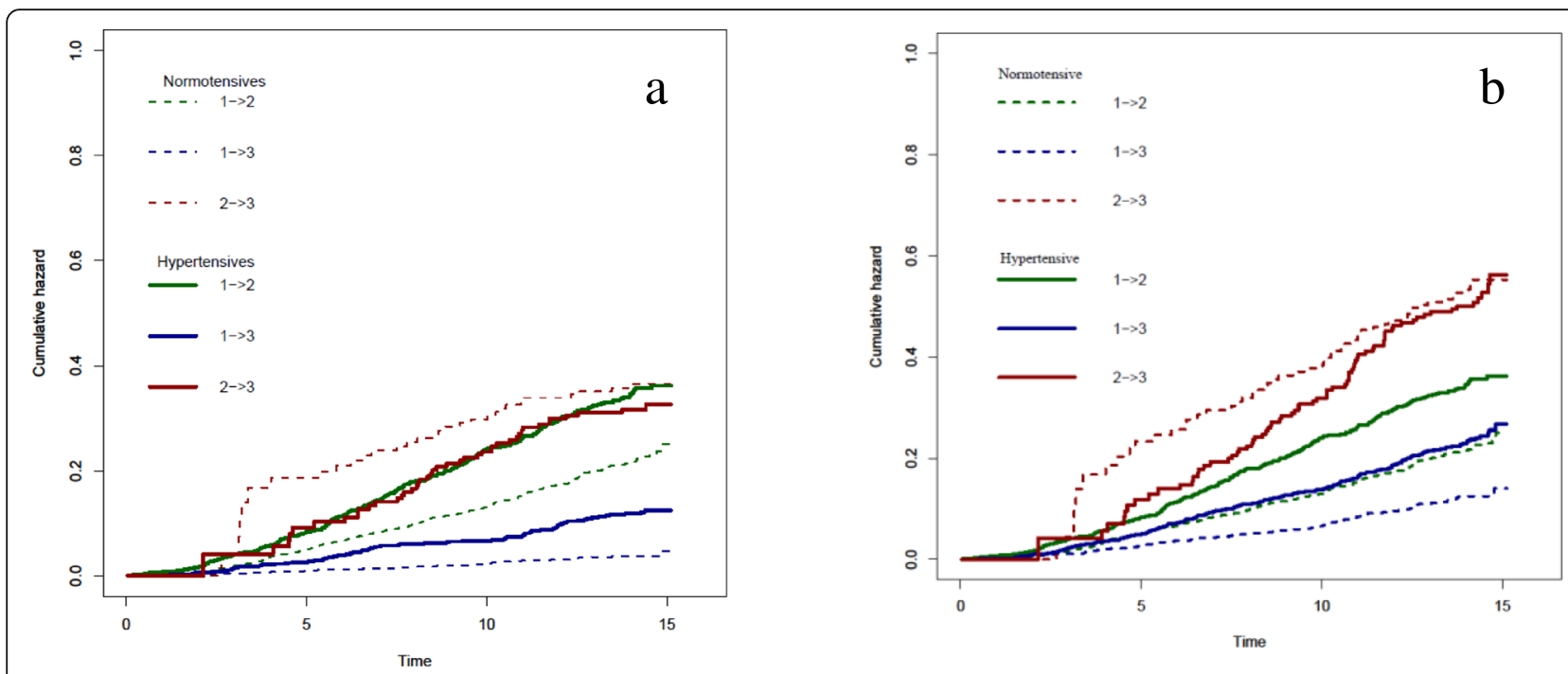

Hypertension was defined according to JNC7 guideline
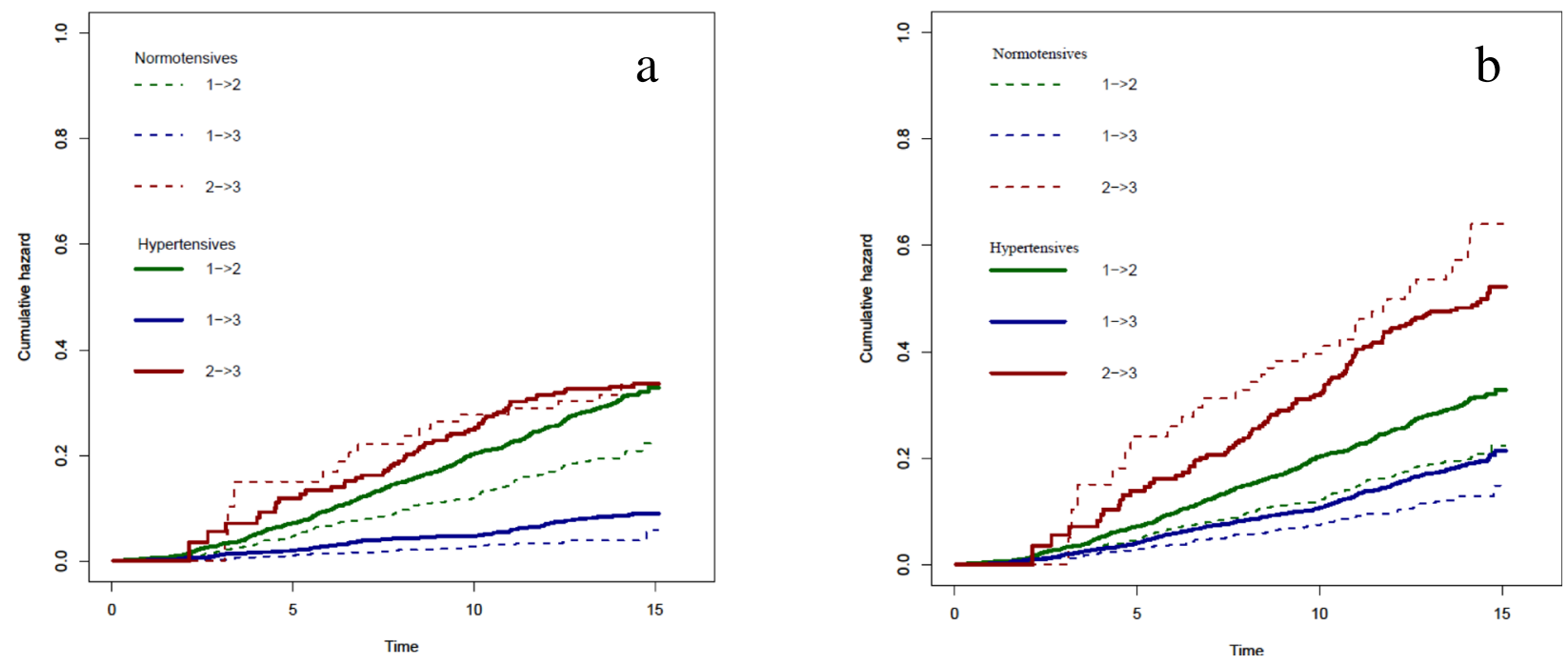

Hypertension was defined according to the 2017 ACC/AHA guideline

Fig. 3 Estimated baseline cumulative hazards, normotensives vs. hypertensives, Tehran lipid and glucose study (1999-2014) a Multi-state model 1 in which the state 3 is CVD death. $\mathbf{b}$ Multi-state model 2 in which the state 3 is all-cause mortality. 1 $\rightarrow$ 2: CVD-free to non-fatal CVD. $1 \rightarrow 3:$ CVDfree to CVD death in A, and CVD-free to all-cause death in B. 2 $\rightarrow$ 3: Non-fatal CVD to CVD death in A, and CVD-free to all-cause death in B

\section{Results of MSM2}

Table 3 shows transition hazards from MSM 2. The results for transition 1 (CVD free to non-fatal CVD) was quite similar to the results of transition 1 in MSM1. In transition 2 , the results of confounders adjusted models showed that hypertension was associated with 64 and $31 \%$ increased risk of all-cause death according to JNC7 and the ACC/AHA guidelines, respectively. The differences between two HRs was significant $(p=0.020)$. In transition 3, we did not find significant difference between hypertensives and normotensives regarding the risk of all-cause mortality after the non-fatal CVD occurrence according to both JNC7 and the 2017 ACC/AHA guidelines (Table 3 ).

Table 4 shows that the LE without CVD were 0.7 and 0.4 years shorter in hypertensives compared to their normotensive counterparts according to JNC7 and the 2017 ACC/AHA guidelines, respectively. Hypertensive participants experienced a first non-fatal CVD about 0.9 and 0.6 years earlier than normotensive population 
Table 3 Estimation of Hazard ratios and confidence intervals of hypertension for non-fatal CVD and CVD death and all-cause death with and without CVD according to JNC7 and the 2017 ACC/AHA guidelines, Tehran Lipid and Glucose Study (1999-2014)

\begin{tabular}{llllll}
\hline Number of participants & Sex and age & Confounders & Number of participants & Sex and age & Confounders \\
entered /number of events & adjusted & adjusted & entered /number of & adjusted & adjusted \\
& HR $(95 \% \mathrm{Cl})$ & $\mathrm{HR}(95 \% \mathrm{Cl})$ & events & $\mathrm{HR}(95 \% \mathrm{Cl})$ & $\mathrm{HR}(95 \% \mathrm{Cl})$ \\
\hline
\end{tabular}

\section{Multi-state model 1}

Transitions According to JNC7

CVD-free to non-fatal CVD

$\begin{array}{ll}\text { Total } & 3002 / 601 \\ \text { Normotensive } & 1701 / 283 \\ \text { Hypertensive } & 1301 / 318\end{array}$

\section{CVD-free to CVD death}

$\begin{array}{ll}\text { Total } & 3002 / 156 \\ \text { Normotensive } & 1701 / 49 \\ \text { Hypertensive } & 1301 / 107\end{array}$

Non-fatal CVD to CVD death

$\begin{array}{ll}\text { Total } & 601 / 71 \\ \text { Normotensive } & \text { 245/30 } \\ \text { Hypertensive } & 356 / 41\end{array}$

\section{Multi-state model 2}

CVD-free to non-fatal CVD

$\begin{array}{ll}\text { Total } & 3002 / 601 \\ \text { Normotensive } & 1701 / 283 \\ \text { Hypertensive } & 1301 / 318\end{array}$

Hypertensive 1301/318

CVD-free to all-cause death CVD-free to CVD death

$\begin{array}{ll}\text { Total } & 3002 / 493 \\ \text { Normotensive } & 1701 / 208 \\ \text { Hypertensive } & 1301 / 285\end{array}$

Non-fatal CVD to all-cause death

$\begin{array}{ll}\text { Total } & 601 / 126 \\ \text { Normotensive } & 245 / 51 \\ \text { Hypertensive } & 356 / 75\end{array}$

In multi-state model 1 transition 3 is CVD death, and in multi-state model 2 transition 3 is all-cause death; Confounders included: age, sex, smoking, total cholesterol, prevalent diabetes and body mass index; CVD: cardiovascular disease; ${ }^{\mathrm{P}} \mathrm{P}$-value $<0.001 ;{ }^{\dagger} \mathrm{P}$-value $<0.01$

according to JNC7 and the 2017 ACC/AHA guidelines, respectively. No significant difference was observed between the LE of two hypertensive and normotensive participants after experiencing a first non-fatal CVD according to both guidelines. The RMTL were 1.9 and 1.5 times higher in hypertensive vs. normotensive participants according to JNC7 and the 2017 ACC/AHA guidelines, respectively (Table 4). The results of adjusted RMST showed that hypertensive individuals had the shorter RMST, in transitions 1 and 2, according to both JNC7 and the 2017 ACC/AHA guidelines (Table 5).

\section{Discussion}

The results of multi-state analysis among CVD-free popu-

lations aged $\geq 50$ years shows that although hypertension is

\section{According to the 2017 ACC/AHA}

$3002 / 601$

$942 / 143$

$2060 / 458$

$3002 / 156$

$942 / 29$

2060/127

$601 / 71$

$142 / 20$

$459 / 51$

$3002 / 601$

$942 / 143$

$2060 / 458$

Reference Reference

$1.55(1.28-1.87)^{*} \quad 1.48(1.21-1.80)^{*}$

$3002 / 493$

$942 / 122$

2060/371

Reference Reference

$1.30(1.02-1.66)^{\dagger} \quad 1.31(1.01-1.69)^{\dagger}$

$601 / 126$

$142 / 31$

$142 / 31$

Reference

Reference

$0.95(0.63-1.43) \quad 1.00(0.66-1.51)$

significantly associated with transition to both CVD and all-cause mortality, using both the 2017 ACC/AHA and JNC7 guidelines, after incident non-fatal CVD (prevalent CVD), hypertension does not appear to have a significant association with either CVD or all-cause mortality according to both above mentioned guidelines. Moreover, hypertensive patients experienced a lower LE free of CVD and more years of life lost as compared to normotensive participants, with attenuation of this effect if a lower BP threshold was used via the 2017 ACC/AHA guidelines as compared to the JNC7. Also, this effect was primarily limited to the transition from CVD-free to either fatal events or non-fatal CVD events, and did not affect on transition from non-fatal CVD states to fatal events, using both guidelines. 


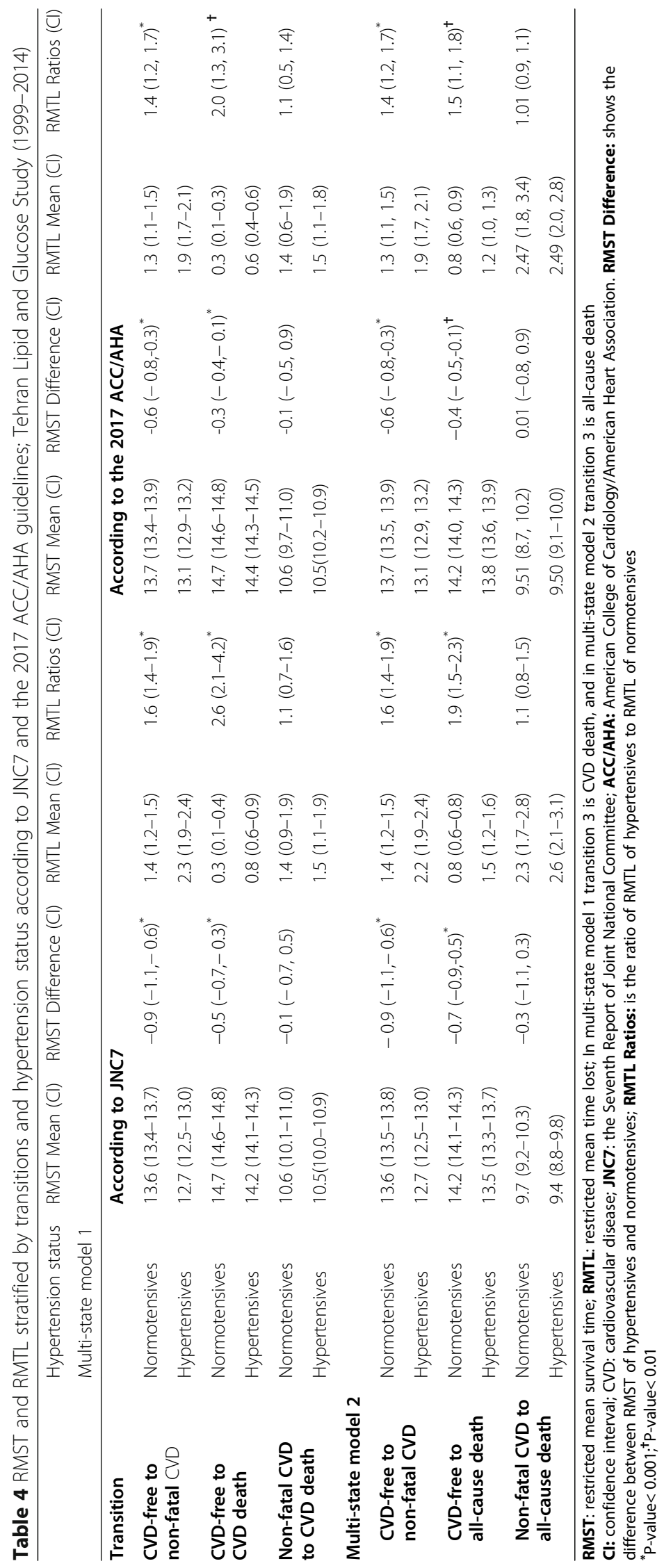


Table 5 Adjusted restricted mean survival time (RMST) of study participants according to JNC7 and the 2017 ACC/AHA guidelines; Tehran Lipid and Glucose Study (1999-2014)

\begin{tabular}{|c|c|c|c|c|c|c|}
\hline & Difference of estimated RMST & SE & P-value & Difference of estimated RMST & SE & P-value \\
\hline & \multicolumn{6}{|l|}{ Multi-state model 1} \\
\hline Transition & \multicolumn{3}{|l|}{ According to JNC7 } & \multicolumn{3}{|c|}{ According to the $2017 \mathrm{ACC} / \mathrm{AHA}$} \\
\hline \multicolumn{7}{|c|}{ CVD-free to non-fatal CVD } \\
\hline Hypertensives & -0.71 & 0.14 & $<0.001$ & -0.5 & 0.14 & $<0.001$ \\
\hline \multicolumn{7}{|c|}{ CVD-free to CVD death } \\
\hline Hypertensives & -0.71 & 0.14 & $<0.001$ & -0.5 & 0.14 & $<0.001$ \\
\hline \multicolumn{7}{|c|}{ Non-fatal CVD to CVD death } \\
\hline Hypertensives & -0.12 & 0.4 & 0.75 & 0.10 & 0.40 & 0.838 \\
\hline \multicolumn{7}{|c|}{ Multi-state model 2} \\
\hline \multicolumn{7}{|c|}{ CVD-free to non-fatal CVD } \\
\hline Hypertensives & -0.71 & 0.14 & $<0.001$ & -0.54 & 0.14 & $<0.001$ \\
\hline \multicolumn{7}{|c|}{ CVD-free to all-cause death } \\
\hline Hypertensives & -0.71 & 0.14 & $<0.001$ & -0.54 & 0.14 & $<0.001$ \\
\hline \multicolumn{7}{|c|}{ Non-fatal CVD to all-cause death } \\
\hline Hypertensives & -0.35 & 0.44 & 0.42 & -0.30 & 0.44 & 0.567 \\
\hline \multicolumn{7}{|c|}{$\begin{array}{l}\text { In multi-state model } 1 \text { transition } 3 \text { is CVD death, and in multi-state model } 2 \text { transition } 3 \text { is all-cause death } \\
\text { SE: standard error; CVD: cardiovascular disease; JNC7: the Seventh Report of Joint National Committee; } \\
\text { ACC/AHA: American College of Cardiology/American Heart Association } \\
\text { Restricted mean survival times (RMST) were estimated using pseudo-value technique } \\
\text { Estimated RMST were adjusted for age, sex, smoking, total cholesterol, prevalent diabetes and body mass index } \\
\text { In multi-state model 1, patients with hypertension had the significantly shorter RMST, namely } 0.71 \text { in transitions } 1 \text { and 2, and } 0.5 \text { in transitions } 1 \text { and } 2 \text { according } \\
\text { to JNC7 and the 2017 ACC/AHA guideline, respectively, than normotensive participants } \\
\text { In multi-state model 2, patients with hypertension had the significantly shorter RMST, namely } 0.71 \text { years in transitions } 1,2 \text { according to JNC7 guideline and } 0.54 \\
\text { years in transitions 1, } 2 \text { according to the } 2017 \text { ACC/AHA guideline, than normotensive participants }\end{array}$} \\
\hline
\end{tabular}

Abundant epidemiological studies have shown the significant associations between hypertension and an increased risk of CVD/all-cause mortality [3, 25, 26]. More recently, we showed that hypertension increased the risks of CVD (HR: 1.89; 95\% CI:1.20-2.98) and all-cause death $(2.01,1.26-3.20)$ among Iranian middle-aged population [3]. A meta-analysis showed that $20 \mathrm{mmHg}$ higher SBP and $10 \mathrm{mmHg}$ higher DBP were each associated with a doubling in the risk of death from CVD [27]. These findings have been confirmed in the present study, which reports 52 and $48 \%$ increased risks of non-fatal CVD according to JNC7 and the 2017 ACC/AHA guidelines, respectively. We also showed that among CVD-free population, hypertension was associated with increased risk of CVD death according to both JNC7 (2.96; 95\% CI $2.06-4.25)$ and the 2017 ACC/AHA $(1.98 ; 1.30-3.04)$ criteria; the corresponding values were $(1.64 ; 1.32-2.05)$ and $(1.31 ; 1.01-1.69)$ for all-cause mortality.

In fact, by lowering the cutoff values of SBP/DBP, the association between BP and mortality events among CVD-free population remained significant but was attenuated. It is quite evident that the more stringent $\mathrm{BP}$ thresholds will markedly increase the number of people classified as having hypertension [28-30]. As nonpharmacological therapy without taking medicines is recommended for all adults with SBP/DBP of 130-139/
$80-90 \mathrm{mmHg}$, excluding those with aged 65 years or older, pre-existing atherosclerotic CVD or a 10 year predicated risk of developing it of $\geq 10 \%$, chronic kidney disease or T2D [12], the diagnosis of hypertension by using ACC/AHA guideline may provide an opportunity for people to change diet and lifestyle habits and to emphasize that BP is a risk factor that can be controlled $[12,31]$.

By using both JNC7 and AHA guidelines, we found no significant difference in the risk of CVD death and all-cause mortality between hypertensive and normotensive population after incident non-fatal CVD. However, using Framingham data in 2005, Franco and colleagues [32] arrived at different conclusions. In a multi-state life table analysis, they showed that hypertension was significantly associated with $29 \%$ increased risk $(1.29 ; 1.10-1.52)$ of death after a nonfatal CVD. The loss of associations in transition 3 in our study might be attributable to the treatment of survivors of CVD by new therapeutic approaches. Also, Franco et al. [32] analysed data from 1948 to early 2000s, whereas we selected participants starting from 1999 and followed them until 2014, when there had been significant improvement in the management of hypertension after the incidence of CVD compared to the period between 1950s through 1980s. A 
number of interventional studies has consistently reported that the modification of potential risk factors [33] and the administration of beta-adrenergicblocking agents [34] and aspirin [35] reduce the risk of adverse cardiovascular events after incident CVD. However, further investigations are needed to clarify and discuss our results.

We found that over 15 years follow-up, in MSM1,potential lifetimes were about 6 and 4 months fewer among CVD-free hypertensive individuals, according to JNC7 and the 2017 ACC/AHA guidelines, respectively, and in comparison to their normotensive participants. The corresponding values were 8.4 and 5 months for MSM2. Although few studies have investigated the impact of hypertension on LE in Western [32, 36] and Asian [37] populations, the effect of hypertension on LE has not been reported in the Eastern Mediterranean region with the high burden of CVD [38]. In the study conducted by Franco et al. [32], it was reported that at 50 years of age, total LE was 5.1 and 4.9 years longer for normotensive males and females, respectively, than for their hypertensive counterparts. In the present study, we found no significant difference in LE with CVD between hypertensive and normotensive individuals in both MSMs. However, Franco et al. [32] showed that hypertensives lived 2 years more with CVD compared with normotensive participants. A reason for the difference between their and our study could regard the different methodology used to calculate LE. While we estimated LE over 15 years of follow up using RMST, Franco et al. estimated total LE using life table analysis. Previous studies have suggested that the prognosis of survivors of non-fatal CVD is related to complex interactions between a number of factors, such as age, coexisting conditions, the extent of coronary artery disease, adjuvant medication use [39] and implementing effective interventions [40].

According to national and cohort studies in Iran, hypertension (defined by JNC7) was highly prevalent in adults [41-43]. In the last national study conducted in Iran, 25.2\% (6.6 million cases) of Iranian people aged between 25 and 64 years had high BP, 45.5\% were prehypertensive, $66 \%$ of hypertensive patients were unaware of their disease, $75 \%$ did not take medication to lower BP and $76 \%$ had their BP uncontrolled [41]. These proportions will dramatically increase under the new ACC/ AHA guidelines. Thus, applying new criteria could have a significant impact on individuals as well as on health care system. Although the new definition tends to increase acute health care costs, these treatments may delay the onset of a non-fatal CVD and incidence of mortality events and consequently other long-term care required.

This is the first multi-state analysis using Markov model which estimates the association between hypertension and mortality considering non-fatal CVD as an intermediate event. However, the results should be interpreted in the context of several limitations. First, due to the small number of CVD events, we were unable to investigate the cause-specific associations separately for each CVD event. Second, details about the treatment approach after incident non-fatal CVD were not available. Lastly, the study was conducted among Iranian population and therefore results might not be generalizable to other country.

\section{Conclusion}

Using the 2017 ACC/AHA guideline increased the prevalence of hypertension in our population. Analyzing multistate data under a time homogeneous semi-Markov model showed that hypertension was associated with increased risk of non-fatal CVD, CVD death and all-cause mortality among CVD-free populations aged $\geq 50$ years, and reduction in the number of years lived without CVD and early onset of CVD, and consequently, an increase in the time spent with these diseases. No significant association was observed between hypertension and mortality events after incident CVD using both JNC7 and 2017 ACC/AHA guidelines.

\section{Abbreviations \\ ACC/AHA: The American College of Cardiology/American Heart Association: BMI: Body mass index; CHD: Coronary heart disease; Cl: Confidence interval; CVD: Cardiovascular disease; DBP: Diastolic blood pressure; FPG: Fasting plasma glucose; HR: Hazard ratio; LE: Life expectancy; MI: Myocardial infarction; MSM: Multi-state model; RMST: Restricted mean survival time; RMTL: Restricted mean time lost; SBP: Systolic blood pressure; TC: Total cholesterol}

\section{Acknowledgements}

This study was conducted in the framework of the Tehran Lipid and Glucose Study (TLGS) and was supported by the Research Institute for Endocrine Sciences, Shahid Beheshti University of Medical Sciences. We express our appreciation to TLGS participants and the research team members for their contribution to the study.

\section{Authors' contributions}

AR performed the data analysis and drafted the manuscript. MJB and MM conceived the whole study and reviewed the manuscript critically. FA and FHS designed the study protocol, and participated in the coordination and management of the study. FH had access to all data for this study and takes responsibility for the manuscript contents. All authors have read and approved the final manuscript.

\section{Funding}

This study was supported by grant No. 121 from the National Research Council of the Islamic Republic of Iran. This funding source had no role in the design of the study and collection, analysis, and interpretation of data and in writing the manuscript.

\section{Availability of data and materials}

All relevant data are included within the paper. Additional data are available upon request to the head of the RIES Ethics Committee, Dr. Azita ZadehVakili (azitavakili@endocrine.ac.ir).

\section{Ethics approval and consent to participate}

The ethics committee of the Research Institute for Endocrine Sciences of Shahid Beheshti University of Medical Sciences approved the study and informed written consent was obtained from all participants. 


\section{Consent for publication}

Not applicable.

\section{Competing interests}

The authors declare that they have no competing interests.

\section{Author details}

'Prevention of Metabolic Disorders Research Center, Research Institute for Endocrine Sciences, Shahid Beheshti University of Medical Sciences, Tehran, Iran. ${ }^{2}$ Ciccarone Center for the Prevention of Heart Disease, Johns Hopkins University School of Medicine, Baltimore, USA. ${ }^{3}$ Endocrine Research Center, Research Institute for Endocrine Sciences, Shahid Beheshti University of Medical Sciences, Tehran, Iran.

Received: 18 September 2019 Accepted: 25 June 2020

Published online: 06 July 2020

\section{References}

1. O'Doherty MG, Cairns K, O'Neill V, et al. Effect of major lifestyle risk factors, independent and jointly, on life expectancy with and without cardiovascular disease: results from the consortium on health and ageing network of cohorts in Europe and the United States (CHANCES). Eur J Epidemiol. 2016;31(5):455-68

2. Bundy JD, Li C, Stuchlik P, et al. Systolic blood pressure reduction and risk of cardiovascular disease and mortality: a systematic review and network meta-analysis. JAMA Cardiol. 2017:2(7):775-81.

3. Lotfaliany M, Akbarpour S, Mozafary A, et al. Hypertension phenotypes and incident cardiovascular disease and mortality events in a decade follow-up of a Middle East cohort. J Hypertens. 2015;33(6):1153-61.

4. Kearney PM, Whelton M, Reynolds K, et al. Global burden of hypertension: analysis of worldwide data. Lancet. 2005;365(9455):217-23.

5. Vega G, Martínez S, Jiménez PA, et al. Effect of cardiovascular risk factors on long-term morbidity and mortality following acute myocardial infarction. Rev Esp Cardiol. (English Edition. 2007;60(7):703-13.

6. Zornoff LA, Paiva SA, Assalin VM, et al. Clinical profile, predictors of mortality, and treatment of patients after myocardial infarction, in an academic medical center hospital. Arq Bras Cardiol. 2002;78(4):401-5.

7. Andersen PK, Keiding N. Multi-state models for event history analysis. Stat Methods Med Res. 2002:11(2):91-115.

8. Meira-Machado L, de Uña-Álvarez J, Cadarso-Suárez C, et al. Multi-state models for the analysis of time-to-event data. Stat Methods Med Res. 2009; 18(2):195-222.

9. Putter H, Fiocco M, Geskus RB. Tutorial in biostatistics: competing risks and multi-state models. Stat Med. 2007:26(11):2389-430.

10. Gasperoni F, leva F, Barbati G, et al. Multi-state modelling of heart failure care path: a population-based investigation from Italy. PLoS One. 2017;12(6): e0179176.

11. Ramezankhani A, Azizi F, Hadaegh F, et al. Diabetes and number of years of life lost with and without cardiovascular disease: a multi-state homogeneous semi-Markov model. Acta Diabetol. 2018:55(3):253-62

12. Whelton PK, Carey RM, Aronow WS, et al. 2017 ACC/AHA/AAPA/ABC/ACPM/ AGS/APhA/ASH/ASPC/NMA/PCNA Guideline for the Prevention, Detection, Evaluation, and Management of High Blood Pressure in Adults: A Report of the American College of Cardiology/American Heart Association Task Force on Clinical Practice Guidelines. J Am Coll Cardiol. 2018;11(19):e127-e248.

13. Chobanian AV, Bakris GL, Black HR, et al. Seventh report of the joint national committee on prevention, detection, evaluation, and treatment of high blood pressure. Hypertension. 2003;42(6):1206-52.

14. Azizi F, Ghanbarian A, Momenan AA, et al. Prevention of noncommunicable disease in a population in nutrition transition: Tehran lipid and glucose study phase II. Trials. 2009;10(1):5

15. Sardarinia M, Akbarpour S, Lotfaliany $M$, et al. Risk factors for incidence of cardiovascular diseases and all-cause mortality in a middle eastern population over a decade follow-up: Tehran lipid and glucose study. PLoS One. 2016;11(12):e0167623.

16. Association AD. Standards of medical care in diabetes-2014. Diabetes Care. 2014;37(Supplement 1):S14-80.

17. Khalili D, Azizi F, Asgari S, et al. Outcomes of a longitudinal populationbased Cohort Study and pragmatic community trial: Findings from 20 years of the Tehran Lipid and Glucose Study. Int J Endocrinol Metab. 2018;16(4 Suppl):e84748
18. Buuren S, Groothuis-Oudshoorn K. mice: Multivariate imputation by chained equations in R. J Stat Softw. 2011:45(3):1-67.

19. Hougaard P. Multi-state models: a review. Lifetime Data Anal. 1999;5(3):239-64.

20. Royston P, Parmar MK. The use of restricted mean survival time to estimate the treatment effect in randomized clinical trials when the proportional hazards assumption is in doubt. Stat Med. 2011;30(19):2409-21.

21. Zhao $L$, Claggett $B$, Tian $L$, et al. On the restricted mean survival time curve in survival analysis. Biometrics. 2016;72(1):215-21.

22. Klein JP, Gerster M, Andersen PK, et al. SAS and R functions to compute pseudo-values for censored data regression. Comput Methods Prog Biomed. 2008;89(3):289-300.

23. De Wreede LC, Fiocco M, Putter $\mathrm{H}$. The mstate package for estimation and prediction in non-and semi-parametric multi-state and competing risks models. Comput Methods Prog Biomed. 2010;99(3):261-74.

24. Schemper M, Smith TL. A note on quantifying follow-up in studies of failure time. Control Clin Trials. 1996;17(4):343-6.

25. Oh J-Y, Allison MA, Barrett-Connor E. Different impacts of hypertension and diabetes mellitus on all-cause and cardiovascular mortality in communitydwelling older adults: the rancho Bernardo study. J Hypertens. 2017;35(1): $55-62$.

26. Ikeda $\mathrm{A}$, Iso $\mathrm{H}$, Yamagishi $\mathrm{K}$, et al. Blood pressure and the risk of stroke, cardiovascular disease, and all-cause mortality among Japanese: the JPHC study. Am J Hypertens. 2009;22(3):273-80.

27. Collaboration PS. Age-specific relevance of usual blood pressure to vascular mortality: a meta-analysis of individual data for one million adults in 61 prospective studies. Lancet. 2002;360(9349):1903-13.

28. Glümer C, Carstensen B, Sandbæk A, et al. A Danish diabetes risk score for targeted screening: the Inter99 study. Diabetes Care. 2004;27(3):727-33.

29. Khera R, Lu Y, Lu J, et al. Impact of 2017 ACC/AHA guidelines on prevalence of hypertension and eligibility for antihypertensive treatment in United States and China: nationally representative cross sectional study. bmj. 2018; 362:k2357.

30. Lee $\mathrm{JH}$, Kim S-H, Kang S-H, et al. Blood pressure control and cardiovascular outcomes: real-world implications of the 2017 ACC/AHA hypertension guideline. Sci Rep. 2018;8(1):1-8.

31. Muntner P, Carey RM, Gidding S, et al. Potential US population impact of the 2017 ACC/AHA high blood pressure guideline. J Am Coll Cardiol. 2018; 71(2):109-18.

32. Franco $\mathrm{OH}$, Peeters $\mathrm{A}$, Bonneux $\mathrm{L}$, et al. Blood pressure in adulthood and life expectancy with cardiovascular disease in men and women. Hypertension. 2005;46(2):280-6.

33. Maessen MF, Eijsvogels TM, Hijmans-Kersten BT, et al. Vascular function and structure in veteran athletes after myocardial infarction. Med Sci Sports Exerc. 2017:49(1):21-8.

34. Borghi C, Omboni S, Reggiardo G, et al. Efficacy of Zofenopril compared with placebo and other angiotensin-converting enzyme inhibitors in patients with acute myocardial infarction and previous cardiovascular risk factors: a pooled individual data analysis of 4 randomized, double-blind, controlled, Prospective Studies. J Cardiovasc Pharmacol. 2017;69(1):48.

35. Anand SS, Bosch J, Eikelboom JW, et al. Rivaroxaban with or without aspirin in patients with stable peripheral or carotid artery disease: an international, randomised, double-blind, placebo-controlled trial. Lancet. 2018;391(10117): 219-29.

36. Jia H, Zack MM, Thompson WW. The effects of diabetes, hypertension, asthma, heart disease, and stroke on quality-adjusted life expectancy. Value Health. 2013:16(1):140-7.

37. Turin TC, Murakami Y, Miura K, et al. Hypertension and life expectancy among Japanese: NIPPON DATA 80. Hypertension Res. 2012;35(9):954-8.

38. Turk-Adawi K, Sarrafzadegan N, Fadhil I, et al. Cardiovascular disease in the Eastern Mediterranean region: epidemiology and risk factor burden. Nat Rev Cardiol. 2018;15(2):106-19.

39. Antonelli L, Katz M, Bacal F, et al. Heart failure with preserved left ventricular ejection fraction in patients with acute myocardial infarction. Arq Bras Cardiol. 2015:105(2):145-50.

40. Kezerashvili A, Marzo K, De Leon J. Beta blocker use after acute myocardial infarction in the patient with normal systolic function: when is it "ok" to discontinue? Curr Cardiol Rev. 2012;8(1):77-84.

41. Esteghamati A, Abbasi M, Alikhani S, et al. Prevalence, awareness, treatment, and risk factors associated with hypertension in the Iranian population: the 
national survey of risk factors for noncommunicable diseases of Iran. Am J Hypertens. 2008;21(6):620-6.

42. Hadaegh F, Hasheminia M, Abdi H, et al. Prehypertension tsunami: a decade follow-up of an Iranian adult population. PLoS One. 2015;10(10):e0139412.

43. Ramezankhani A, Azizi F, Hadaegh F, et al. Sex-specific clustering of metabolic risk factors and their association with incident cardiovascular diseases: a population-based prospective study. Atherosclerosis. 2017;263: 249-56.

\section{Publisher's Note}

Springer Nature remains neutral with regard to jurisdictional claims in published maps and institutional affiliations.

Ready to submit your research? Choose BMC and benefit from:

- fast, convenient online submission

- thorough peer review by experienced researchers in your field

- rapid publication on acceptance

- support for research data, including large and complex data types

- gold Open Access which fosters wider collaboration and increased citations

- maximum visibility for your research: over $100 \mathrm{M}$ website views per year

At $\mathrm{BMC}$, research is always in progress.

Learn more biomedcentral.com/submissions 